\title{
Effect of Chronic Hypoxia on Autonomic Nervous System of Fetal Mice
}

\author{
Ahsan H Khandoker ${ }^{1,2}$, Thuraia Al Khoori ${ }^{1}$, Takuya Ito $^{3}$, Takahiro Minato ${ }^{4}$, Yoshitaka Kimura ${ }^{4}$ \\ ${ }^{1}$ Khalifa University, Abu Dhabi, UAE \\ ${ }^{2}$ The University of Melbourne, Parkville, Australia \\ ${ }^{3}$ Jichi Medical University, Japan \\ ${ }^{4}$ Tohoku University, Sendai, Japan
}

\begin{abstract}
The aim of this study is to investigate how chronic hypoxia could affect the autonomic nervous system in fetal mouse (at gestational day 17.5) by using an invasive Fetal Electrocardiography (FECG) technique. Total of 33 pregnant female mice were divided into two groups (sham control $(N=13)$ and chronic hypoxia $(N=20))$. In chronic hypoxia mice at day 15.5, ligations were placed around the uterine vessels that were tied with nylon thread $0.5 \mathrm{~mm}$ in diameter to induce partially chronic hypoxia. Sham mice were operated on in a fashion similarly but without ligation. On 17.5-day beat-to-beat heart intervals were measured for 20 minutes by using an invasive FECG techniques inserting two needle electrodes and a ground. Results show that tone decreases when entropy increases in sham group. On the other hand in chronic hypoxia group tone increases when entropy increases. Therefore, effect of chronic hypoxia is shown to have more pronounced on tone values in the higher range of entropy values (4.2 6.0). Differences in hypoxic effect on individual fetal mice might represent the differences in maturating sympathetic nervous system because chronic hypoxia induces more sympathetic influences in fetal mice.
\end{abstract}

\section{Introduction}

Chronic placental insufficiency such as hypoxia was reported to cause abnormal brain development with neurological disorders such as cerebral palsy, schizophrenia, and cognitive deficits [1]. Mouse models with genetic manipulations are frequently used to study embryonic development to understand how maternal factors influences fetal development [2]. Since humans and mice use similar genes during development, physiologists get valuable information from a mouse model about how an embryo and its placenta develop over time. Compared to a human pregnancy lasting nine months, a mouse fetus develops quickly, taking only three weeks to get from a one-cell embryo to a fully-grown mouse [3]. In another study [4] the acutely anesthetized pregnant mouse was found to be an excellent model for the experimental investigation of hemodynamic coupling between the developing embryo, maturing placenta, and maternal environment [4].

In most of the recent studies in mouse embryos, ultrasonic methods such as 2D echocardiography, Mmode, and pulsed wave Doppler have matured into valuable tools were used to analyze the cardiovascular system of the adult mouse [5] and the developing embryo [6;7]. Large number of mouse models with congenital heart defects, many of which result in early embryonic mortality [8], has resulted in a need for new technologies that allow the study of cardiac form and function in utero in mouse embryos.

The first organ to develop and function in embryo is the heart. Because of the availability of genetic variants, either natural or transgenic constructs, embryonic mouse nervous system is an ideal model to understand the functioning of autonomic nervous system. Beat to beat hear rate variability from ECG signal provides a noninvasive way to monitor the activity of autonomic nervous system [9].

In our previous paper [10], we demonstrated the procedure to monitor and follow cardiac autonomic nervous system development in utero by Electrocardiography approach. However, there have been no studies to look at how chronic hypoxia affects the autonomic nervous system. Therefore the aim of this study is to develop a fetal Electrocardiography system to look at how cardiac autonomic nervous system is affected by chronic hypoxia to fetal mice.

\section{Methods}

\subsection{Mice and samples}

The experiments in this study were conducted in accordance with the Tohoku University guidelines for animal experimentation. The experimental protocol in this study was approved by the Tohoku University Committee 
for Safety Management of Animals. Total of 33 female mice of type C57BL/6N with body weight of 18-22g (914 weeks) were used in this study. Out of them 20 fetuses were induced chronic hypoxia and 13 cases were sham undergoing only surgery without hypoxia being induced. Mice were purchased from CLEA Japan Inc and housed in cages under control lighting of 12:12h light-dark cycle and temperature of $(24 \mathrm{C})$.

\subsection{Animal preparation and surgery}

Mice were bred overnight marking gestation day 0 (GD 0 ) under specific pathogen-free conditions at the Animal Research Institute of Tohoku University in Japan. On the day 12 subcutaneous ketamine (Ketalar 500 mg DaiichiSankyo: $100 \mathrm{mg} / \mathrm{Kg}$ ) and xylazine (ROMPUN INJ. SOLUTION $2 \%$ Bayer; $10 \mathrm{mg} / \mathrm{Kg}$ ) were used to anesthetize the mice and maintained with inhalational isoflurane (Forane AbbVie Inc.: 0.5\%, $260 \mathrm{ml} / \mathrm{min}$ ). Temperature and humidity were controlled at $36.5 \pm 1^{\circ} \mathrm{C}$ and $65 \pm 10 \%$, respectively, during the surgery. Maternal abdomen was depilated with commercial hair removal cream (Veet, Reckitt Benckiser Group plc, Slough, England, UK), and this was followed by an abdominal midline incision. The maternal uterine horns containing 2-8 fetuses were exposed on day 15.5 gestation. Ligation of uterine artery was performed by partially occluding the uterine artery with nylon wire. Hypoxic condition was measured by partial $\mathrm{O} 2$ pressure in placental tissue $\mathrm{PO} 2$ Monitor (POG-203: Unique Medical Co.,Ltd. Tokyo, Japan, $\quad \mathrm{PO} 2=20 \pm 3.1 \mathrm{mmHg}$ for chronic hypoxic condition). In the sham control group cases, no occlusion was done $(\mathrm{PO} 2=28 \pm 7.5 \mathrm{mmHg})$.

\subsection{Fetal Electrocardiography(ECG)}

On day 17.5 of gestation ECG signals of pregnant mice were recorded using biomedical amplifier and recording system (Polymate AP1532®:TEAC, Tokyo, Japan).Starting with laparotomy to expose both uterine horns and once heart rate was in stable condition, ECG measurement was started (figure 1) by using fetal electrocardiography (FECG) [11;12]. FECG in the International Patent Application Number: PCT/JP2006/316386 and Clip-release cycles in the Japanese Patent Application Number 2009/176683. In mice, reassuring fetal status was suggested by a fetal heart rate variability ranging between $120-250$ beats per minute (bpm). Fetal heart rate (FHR) was noted to vary according to the fetal number.

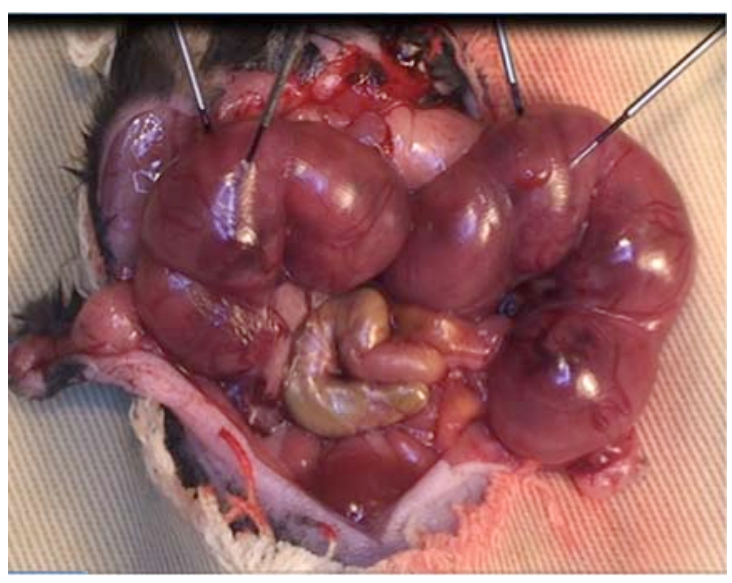

Figure 1. Electrocardiography measurement form pregnant mice underwent surgery on day 17.5 of gestation to monitor the heart rates of both the mother and the fetuses.

\subsection{Fetal HRV indices and Statistics}

\section{Tone-Entropy(TE) Features}

Let, $\mathrm{HR}_{\mathrm{i}}$ represents the series corresponding to any of heart rate and defined as following

$$
\mathrm{HR}=\left\{\mathrm{HR}_{1}, \mathrm{HR}_{2}, H R, \ldots \ldots, \mathrm{HR}_{\mathrm{N}}\right\}
$$

where $\mathrm{N}$ is the total number of beats considered for the analysis (i.e., $\mathrm{N}=400$ ).

In TE analysis, the percentile changes as beat-to-beat heart rate are calculated after normalization by the previous beat as follows

$$
\mathrm{PI}_{\mathrm{i}}=\frac{\mathrm{HR}_{\mathrm{i}}-\mathrm{HR}_{\mathrm{i}+1}}{\mathrm{HR}_{\mathrm{i}}} \times 100
$$

There are increased heart rate if $\mathrm{HR}_{\mathrm{i}+1}>\mathrm{HR}_{\mathrm{i}}$.

Tone is defined as a first order moment (arithmetic average) of the PI time series as

$$
\text { Tone }=\frac{1}{\mathrm{~N}-1} \sum_{\mathrm{i}=1}^{\mathrm{N}-1} \mathrm{PI}_{\mathrm{i}}
$$

Entropy is defined from the probability distribution of PI series by using Shannon's formula

$$
\text { Entropy }=-\sum_{\mathrm{i}=1}^{\mathrm{n}} \mathrm{p}(\mathrm{i}) \log _{2} \mathrm{p}(\mathrm{i})
$$

where $\mathrm{p}(\mathrm{i})$ is a probability of PI having values in the range $\mathrm{i}<\mathrm{PI}<\mathrm{i}+1$, where $\mathrm{i}$ is an integer and $\mathrm{n}$ is the number of bin where, $p(i) \neq 0$. Fig. 1 demonstrates the step-by-step calculation of Tone and Entropy values from heart rate signals.

\section{Results and Discussion}

Figures 2 \& 3 show four examples of beat-by-beat 
fetal heart rates and the histogram profiles estimated from 2 cases in sham group and 2 cases in chronic hypoxia group. In Fig 2, the ranges of PI are different between sham and chronic hypoxia cases resulting in differences in entropy values as shown in Fig 4. On the other hand, in Fig 3, the ranges of PI are not difference the sham and chronic hypoxia cases but portions of positive changes were higher in chronic hypoxia case (Fig. 3 B) resulting in differences in Tone values between the two groups as shown in Fig 4. (A)
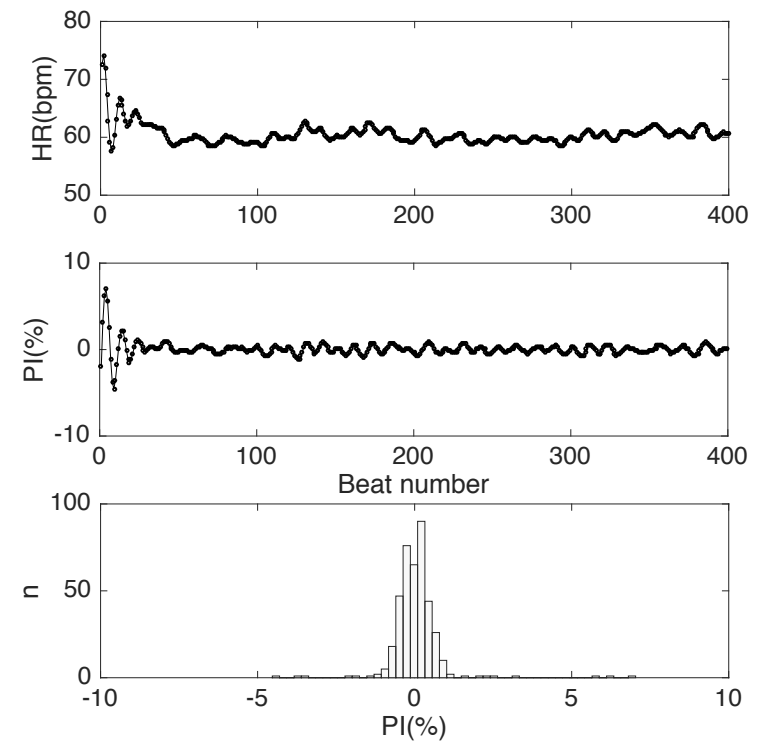

(B)
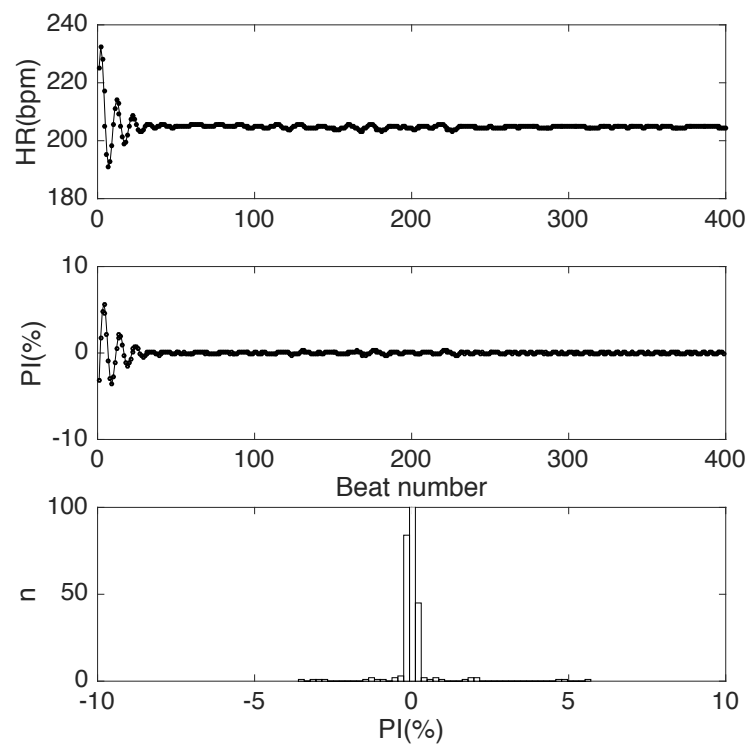

Fig.2. Fetal heart rate (HR), Percentage Index (PI) and Histogram of PI series extracted from one example of sham group (A) and chronic hypoxia group (B) who did not show any significant changes in Tone values.
(A)
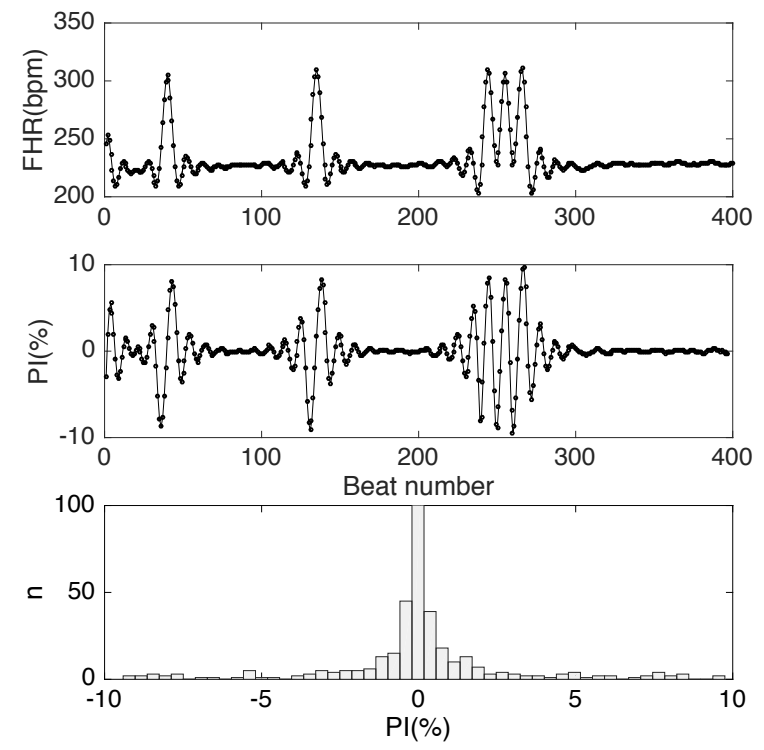

(B)
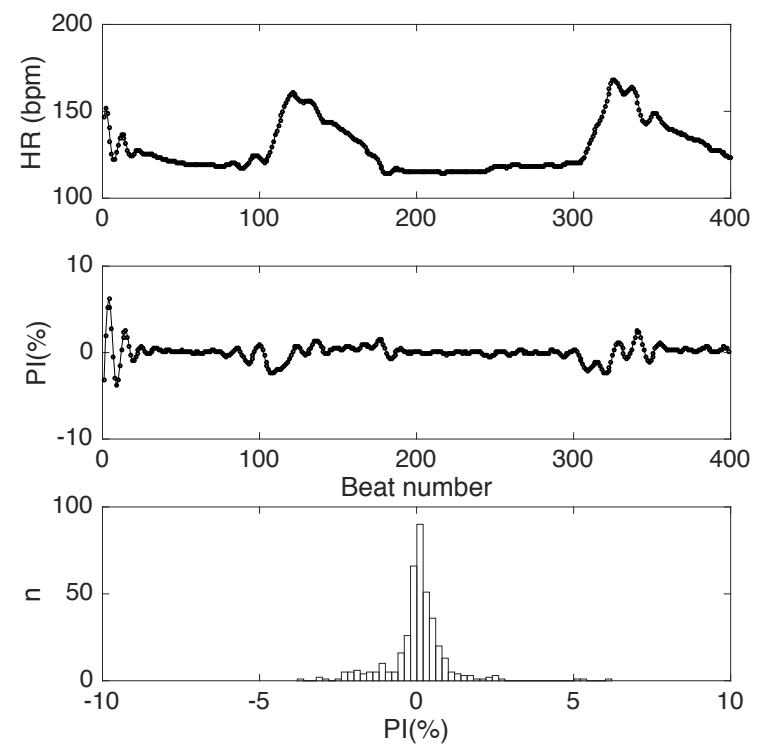

Fig. 3. Fetal heart rate (HR), Percentage Index (PI) and Histogram of PI series extracted from one example of sham group (A) and chronic hypoxia group (B) who did not show any significant changes in Entropy values.

Figure 4 shows the tone entropy values calculated from all the fetuses used in this experiment. It is evident that there are two types of responses to chronic hypoxia in tone entropy of fetal heart rates. 


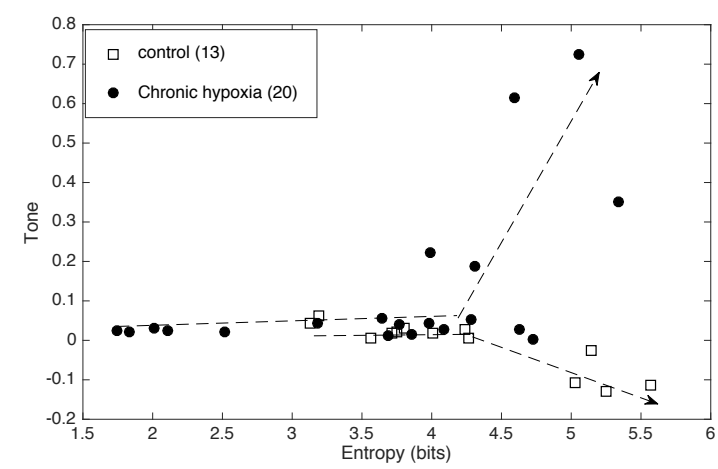

Fig 4. Tone Entropy plot of fetal heart rates of sham control and chronic hypoxia groups at day 17.5.

Table 1: Mean \pm SD of mean fetal heart rate (HR), Standatrd deviation (SD) of HR, Root mean squared successive difference (RMSSD) of HR, Tone and Entropy of HR.

\begin{tabular}{lccc} 
Features & Control (sham) & Chronic Hypoxia & p-values \\
\hline Mean HR & $180.25 \pm 102.22$ & $189.65 \pm 92.16$ & 0.59 \\
SD HR & $31.10 \pm 55.94$ & $33.75 \pm 69.04$ & 0.77 \\
RMSSD HR & $14.11 \pm 31.98$ & $14.35 \pm 35.33$ & 0.47 \\
Tone & $-0.01 \pm 0.06$ & $0.11 \pm 0.22$ & 0.04 \\
Entropy & $3.67 \pm 0.95$ & $3.55 \pm 1.09$ & 0.56
\end{tabular}

Results show that tone decreases when entropy increases in sham group. On the other hand in chronic hypoxia group tone increases when entropy increases (Fig 4). Therefore, effect of chronic hypoxia is shown to have more pronounced on tone values in the higher range of entropy values (4.2 6.0).

Table I summarizes the mean \pm SD values of mean HR, SD HR, RMSSD HR, Tone and Entropy values of HR from both groups. Statistically ( $<0.05$; rank sum U test), group difference was only found in Tone values.

Percentile change of the successive heart rates with respect to the previous heart rate is expressed as the percentage index (PI). Tone is the balance between accelerations $(\mathrm{PI}>0)$ and inhibitions $(\mathrm{PI}<0)$ of the heart rate and represents the sympatho-vagal balance [11]. The entropy evaluates total acceleration-inhibition activities. Higher value of tone means sympatho-vagal balance is shifted more to heart rate accelerations representing more sympathetic influences. On the other hand, higher entropy reflects more influence from parasympathetic nervous system. From these results it could be speculated that chronic hypoxia induces more sympathetic influences in fetal mice with higher tone values as compared to control group when entropy is higher that 4.2.

In summary, the ability of fetal ECG to assess fetal heart rate and fetal heart rate variability in fetal mice undergoing chronic hypoxia in the last half of pregnancy makes it a valuable tool for neurodevelopmental research.

\section{Acknowledgements}

This study was supported by a Khalifa University Internal grant awarded to A.H. Khandoker.

\section{References}

[1] Neerhof MG, Thaete LG: The fetal response to chronic placental insufficiency. Semin Perinatol. 2008, 32 (3): 201-205

[2] M .Kaufman. "The Atlas of Mouse Development", in Academic Press, New York, 1992.

[3] N.A "Stunning pictures of a mouse fetus from the very start of life", mirror website. [Online].Available: http://www.mirror.co.uk/news/uk-news/stunning-pictures-of-amouse-fetus-from-the-very-658001. [Accessed: March. 13, 2016].

[4] B . Keller, B. Maclennan M. J, Tinney J. P., Yoshigi M. "In vivo assessment of embryonic cardiovascular dimensions and function in day-10.5 to -14.5 mouse embryos", Circ. Res.1996. vol. 79, pp. 247-255.

[5] Y. Q. Zhou, F. S. Foster, B. J. Nieman, L. Davidson, X. J. Chen, and R. M. Henkelman, "Comprehensive transthoracic cardiac imaging in mice using ultrasound biomicroscopy with anatomical confirmation by magnetic resonance imaging", Physiol. Genomics, vol. 18, pp. 232- 244, 2004.

[6] J. Mu, J. C. Slevin, D. Qu, S. McCormick, and S. L. Adamson, "In vivo quantification of embryonic and placental growth during gestation in mice using micro-ultrasound," Reprod. Biol. Endocrinol, vol. 6, p. 34, 2008.

[7] C. K. Phoon and D. H. Turnbull, "Ultrasound biomicroscopy-Doppler in mouse cardiovascular development," Physiol. Genomics, vol. 14, pp. 3-15, 2003.

[8] J. Rossant . "Mouse mutants and cardiac development: new molecular insights into cardiogenesis". Circ Res. 1996; vol. 78, pp. $349-353$.

[9] Task Force, "Heart Rate Variability Standards of Measurement, Physiological Interpretation, and Clinical Use". Circulation. 1996; 93: 1043-1065

[10] Khandoker, Ahsan H., Thuraia Al Khoori, Takuya Ito, Rika Sugibayashi, and Yoshitaka Kimura. "Assessment of autonomic neurodevelopment in the mouse fetuses by using fetal electrocardiography." In Engineering in Medicine and Biology Society (EMBC), 2016 IEEE 38th Annual International Conference of the, pp. 2954-2957. IEEE, 2016

[11] Khandoker AH, Jelinek HF, Moritani T, Palaniswami M. Association of cardiac autonomic neuropathy with alteration of sympatho-vagal balance through heart rate variability analysis. Medical engineering \& physics. 2010 Mar 31;32(2):161-7.

Address for correspondence.

Name. Dr Ahsan Khandoker

Full postal address. Department of Biomedical Engineering, Khalifa University, Abu Dhabi, PO Box 127788, UAE.

E-mail address (optional). ahsan.khandoker@kustar.ac.ae 